\title{
Normalization in 3D PET: Dependence on the Activity Distribution of the Source
}

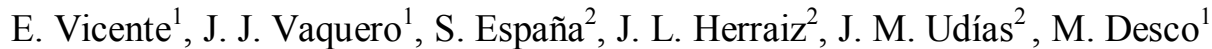 \\ ${ }^{1}$ Unidad de Medicina y Cirugía Experimental, Hospital GU Gregorio Marañón, Madrid, Spain \\ ${ }^{2}$ Dpto. Física Atómica, Molecular y Nuclear, Universidad Complutense, Madrid, Spain
}

\begin{abstract}
In this communication we show the dependence of the normalization of 3D small animal PET scanners on the calibrating source geometry and non-uniformity of the activity distribution inside it. The potential impact on the reconstructed images of deviations from the ideal activity is discussed.

Results from simulated and real data for a pair of block detectors suggest that planar or annular sources can be equally suitable for a normalization procedure if their geometry and their intersections with the system lines-of-response (LORs) are properly taken into account. When comparing an annulus with a centered planar source, both filled with the same total activity, the planar source produces twice the count rate with a $92 \%$ of trues versus $66 \%$ of trues for the annulus. Scatter and randoms are lower by factors of 3 and 4 respectively in the planar case.

We conclude that, although planar and annular sources can be suitable for a normalization procedure if their geometries are properly taken into account, planar source normalization results are better than those of an annulus, particularly if the normalization does not consider the effect of scatter correction, more significant for the annulus source than for the planar one.
\end{abstract}

\section{INTRODUCTION}

M OST normalization methods in PET imaging are based on the assumption that the activity source employed for evaluating crystal and detector efficiencies is completely uniform and perfectly placed in a know location. This assumption could be unrealistic due to possible small misspositioning of the source, inhomogeneities in the activity distribution, or sub-optimal geometry of the source. Accurate normalization in three-dimensional (3D) PET is essential to obtain reconstructed images with high quality and useful quantitative information. Inaccuracies in the normalization method may result in artifacts, poor uniformity and increased noise in the images. Traditional solutions to the calibration problem use models where normalization factors are estimated via direct [1], component-based [2]-[4] or iterative methods

Manuscript received November 17, 2006. E. Vicente: evicente@mce hggm.es, J.J. Vaquero: juanjo@dns.hggm.es, S. España: samuel@nucl.fis.ucm.es, J.L. Herraiz: joaquin@nuc1 fis.ucm.es, J.M. Udias: jose@nuc2.fis.ucm.es, M. Desco: desco@mce.hggm.es

This work has been partially funded by projects PI052583 and PI052583 (Ministerio de Sanidad), BFM2003-04147-C0201 (Ministerio de Edcucación y Ciencia) and CD-TEAM (Ministerio de Industria, CENIT program), Spain.
[5], [6]. Most of them assume that the normalization source has a known geometry and is uniform and perfectly located. In realistic situations this requisite may not be fulfilled.

In this article we study the effects that different deviations from this assumption may have on the calibration process and the quality of the reconstructed images.

\section{MATERIALS AND METHODS}

With the purpose of studying the effect of the geometry of the source phantoms, we made use of Monte Carlo Simulations as well as of real data acquisitions. We evaluated the impact of the geometry of the normalization phantom on the accuracy of the crystal efficiency estimation applied to a pair of real PET detectors block detectors of the rPET scanner (SUINSA Medical Systems, Madrid).

\section{A. Monte Carlo Simulations}

Simulations were performed using the open source GATE [6] software package. GATE, the Geant4 Application for Tomographic Emission, has been proved to be a useful and trustful tool for Monte Carlo simulations. In particular, it allows us to define different sources and detector configurations.

A planar and an annulus sources were simulated. Figures 1 and 2 show the dimensions of both phantoms respectively.

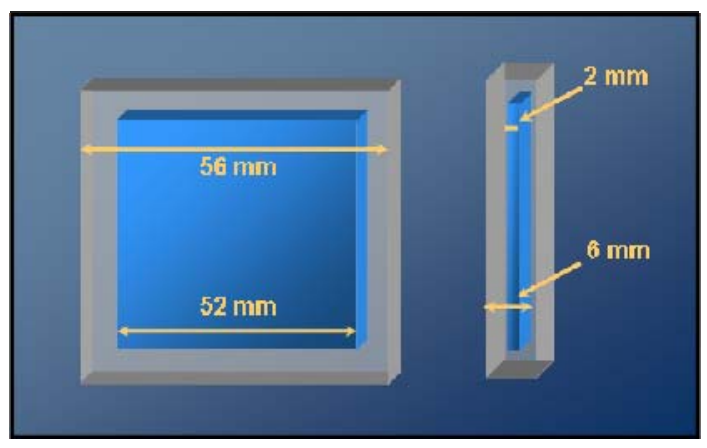

Fig. 1. Planar phantom diagram. The activity distribution is depicted in blue. 


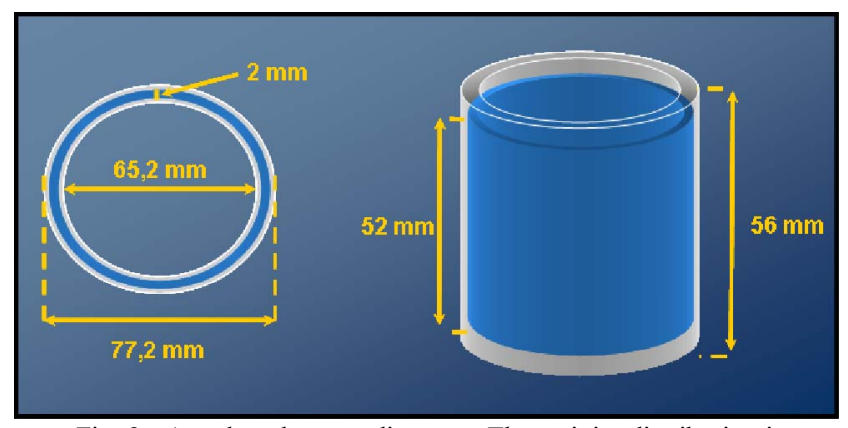

Fig. 2. Annulus phantom diagram. The activity distribution is depicted in blue.

\section{B. Real data}

Real data were acquired with the high-resolution smallanimal rPET [5] scanner (SUINSA Medical Systems, Madrid). This system has four detectors arranged as two orthogonal pairs which rotate $180^{\circ}$. Each detector is comprised of a $30 \times 30$ MLS crystal array $\left(1.5 \times 1.5 \times 12 \mathrm{~mm}^{3}\right)$ optically coupled to a Hamamatsu H8500 flat-panel PS-PMT.

The scanner has a ring diameter of $160 \mathrm{~mm}$ with effective transverse and axial field-of-view of $44.8 \mathrm{~mm}$. The central point sensitivity at the center of the field of view (cFOV) is $2,1 \%(762,2 \mathrm{cps} / \mu \mathrm{Ci})$, the volumetric spatial resolution (cFOV) is $3,4 \mathrm{~mm}^{3}$ and the average energy resolution is $17 \%$.

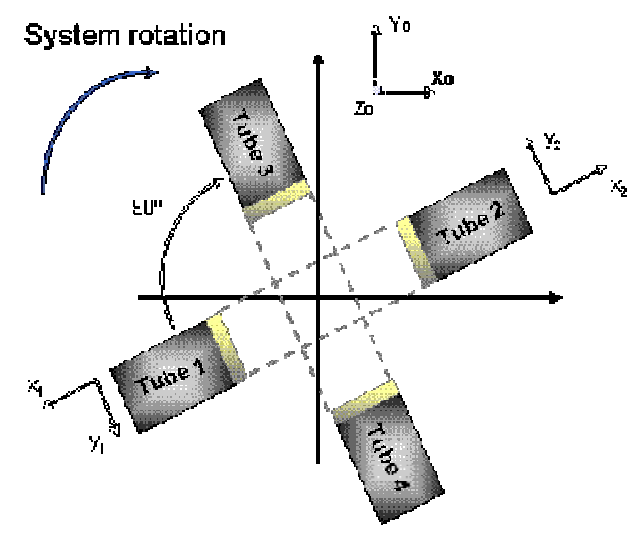

Fig. 3. rPET diagram

Typical activities of the sources for these studies were about $400 \mu \mathrm{Ci}$.

\section{Analysis}

In order to assess the behavior of the FDG planar source and the FDG annulus source, count rate and true, scatter and random coincidences fraction were determined in simulation data.

Real and simulated data were histogrammed in sinograms. Their radial uniformity was examined, correcting the result by the triangular profile that results from the calculations of the number of LORs corresponding to each sinogram bin. In the case of the annulus phantom it is necessary to study its geometry to consider the different contribution from the amount of activity seen by each LOR (see Appendix).

In the analysis of real data, annulus profiles were corrected by two methods: 1) theoretical crosswire length geometric correction, 2) according to simulated annulus. Furthermore, in order to compare the effect of planar and annulus normalizations, a Hot Derenzo (acquired with a pair of rPET detectors) was reconstructed.

\section{RESUltS}

\section{A. Monte Carlo Simulations}

Table 1 shows the simulated results of count fractions (true, scatter and randoms) and count rate for an initial activity of $400 \mu \mathrm{Ci}$ in both phantoms.

TABLE I

SIMULATED COUNT FRACTION AND COUNT RATE OF PLANAR AND ANNULUS FDG SOURCES

\begin{tabular}{c||cccc} 
& $\begin{array}{c}\text { Trues } \\
(\%)\end{array}$ & Scatter (\%) & $\begin{array}{c}\text { Randoms } \\
\mathbf{( \% )}\end{array}$ & $\begin{array}{c}\text { Prompts } \\
\text { (Kcps) }\end{array}$ \\
\hline \hline \multirow{2}{*}{ Planar } & 91,9 & 2,6 & 5,5 & 34,3 \\
Annulus & 66,2 & 9,8 & 24 & 18,8
\end{tabular}

Fig. 4 represents radial profiles on sinogram. Annulus profile was determined with and without the crosswire length geometric correction.

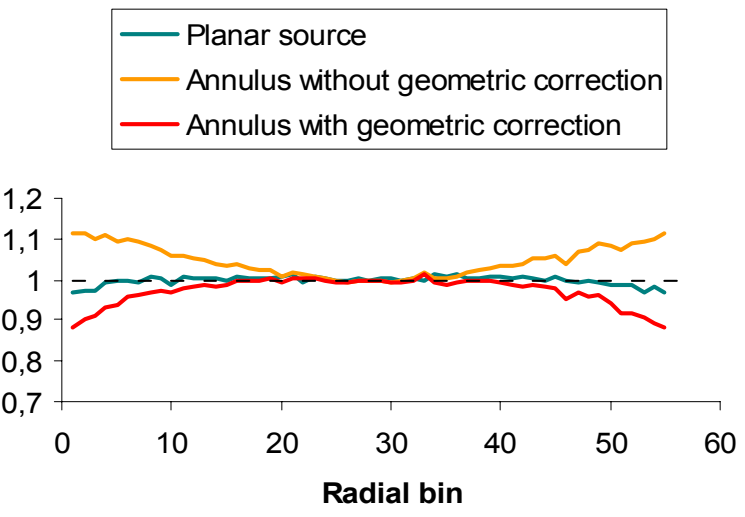

Fig. 4. Radial profiles of simulated planar FDG source and FDG annulus source sinogram.

Fig. 5 compares the profiles of simulated annulus with and without scatter. 


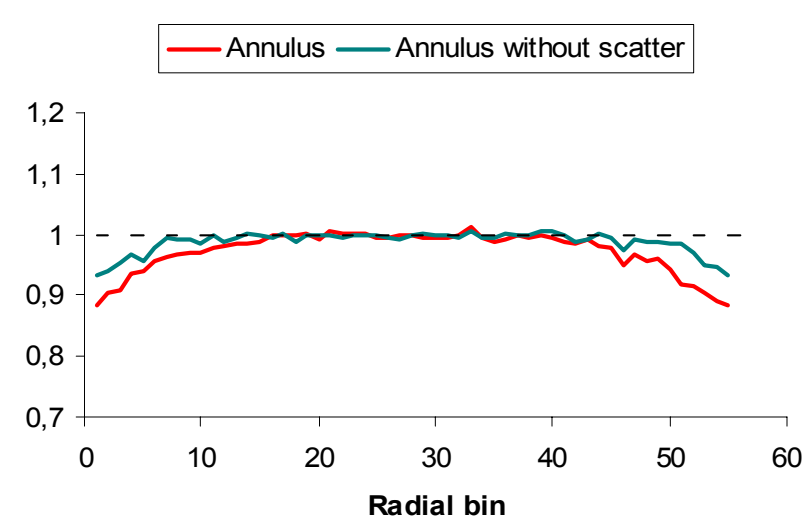

Fig.5. Radial profiles of simulated FDG annulus source sinogram, with (red line) and without scatter (green line).

\section{B. Real data}

Fig. 6 shows the radial profiles on sinogram obtained for real data acquired with a pair of rPET detectors.

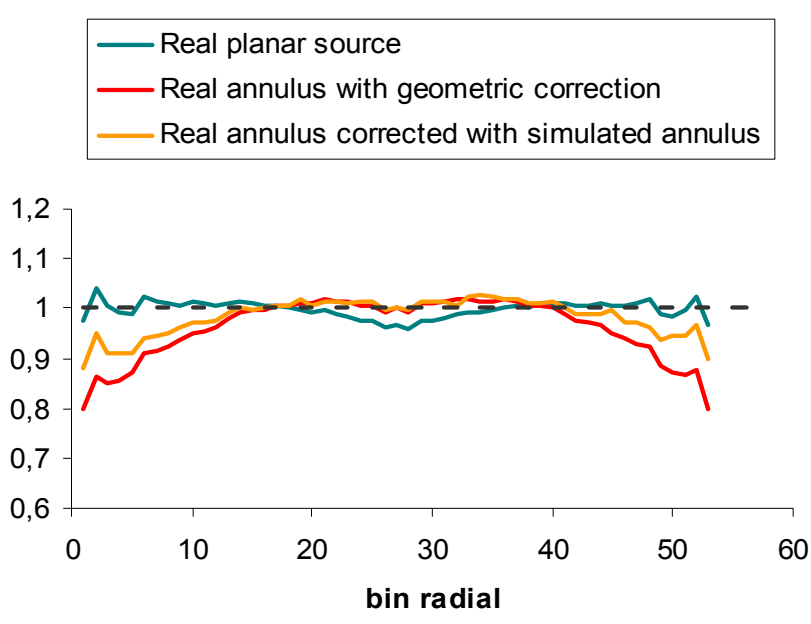

Fig. 6. Radial profiles of real planar fdg source and fdg annulus source sinogram.

Fig. 7 shows one slice of the reconstructed Hot Derenzo images using planar and annulus normalizations. A profile drawn on these images is depicted in Fig. 8.
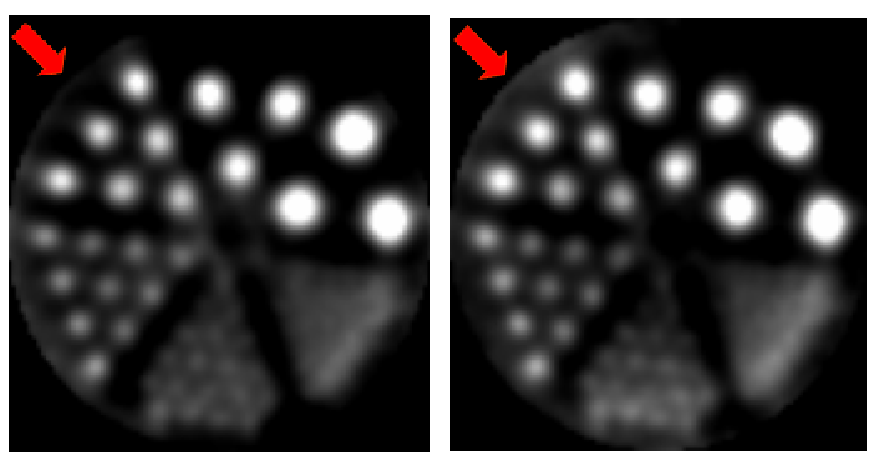

Fig. 7. Hot Derenzo with Planar source (left) and annulus source (rigth) normalization (2D-OSEM reconstruction, 2 iter., 10 subsets.

(Note: reconstructions do not include scatter and randoms corrections).
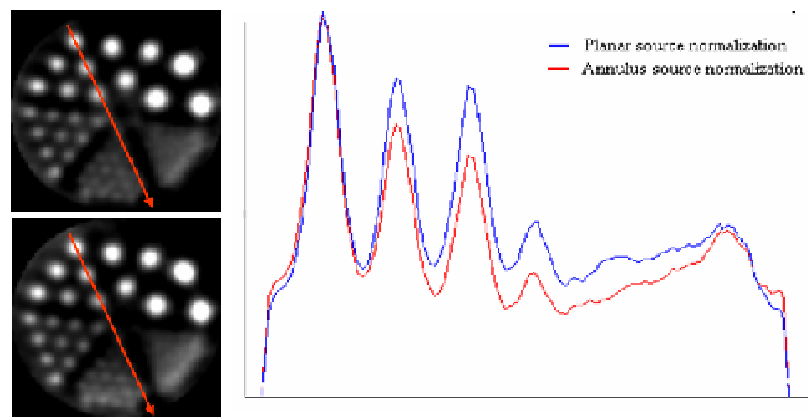

Fig. 8. Radial profile on the reconstructed image with planar and annulus source normalization.

\section{Discussion AND Conclusions}

We can observe in Table 1 that, with the same total activity, the planar source yields higher count rate and number of true coincidences and lower scatter and random coincidences. Therefore, to obtain a similar behavior with the annulus source, it would be necessary to use higher activity or higher acquisition time, being this second option more recommended since it prevents the increase of random coincidences.

In Fig. 4 we can see that the use of geometric correction for simulated annulus sinogram flattens the radial profile, but deviations at the edges are still noticeable. This effect is partly due to scatter coincidences, as we can see in Fig. 5.

Real data profiles shown in Fig 6 indicate that the planar source produces a best normalization than the annulus one. We can also observe that in the case of the annulus normalization, the correction according to simulated annulus is better, but the maximum deviation is still of $10 \%$ at the edges. This edge effect is noticeable in the profiles from the Hot Derenzo images (Fig. 8). We can also see how this effect amplifies the noise at the edges of the image (red arrow, Fig. 7). 
In conclusion, initial results from simulated and real data for a pair of block detectors suggest that planar or annular sources can be equally suitable for a normalization procedure if their geometries are properly taken into account. Nevertheless, planar source normalization yields better results than those obtained when the normalization is made using the annulus source, particularly if the normalization does not consider the effect of scatter correction, more significant for the annulus source than for the planar one.

\section{APPENDIX:}

\section{GEOMETRIC CORRECTION FOR ANNULUS SOURCE}

In the case of the annulus phantom it is necessary to study its geometry in order to consider the different contribution from the amount of activity actually seen by each LOR. As we can see in Fig. 9 (left), the geometric correction for a plane perpendicular to $\mathrm{z}$-axis is:

$$
\begin{aligned}
& X^{2}+Y^{2}=R^{2} \\
& X=x_{0}, \quad Y= \pm\left(R^{2}-x_{0}^{2}\right) \\
& d=\left(R_{2}^{2}-x_{0}^{2}\right)-\left(R_{1}^{2}-x_{0}^{2}\right)
\end{aligned}
$$

Crosswise length $=2 \mathrm{~d}$

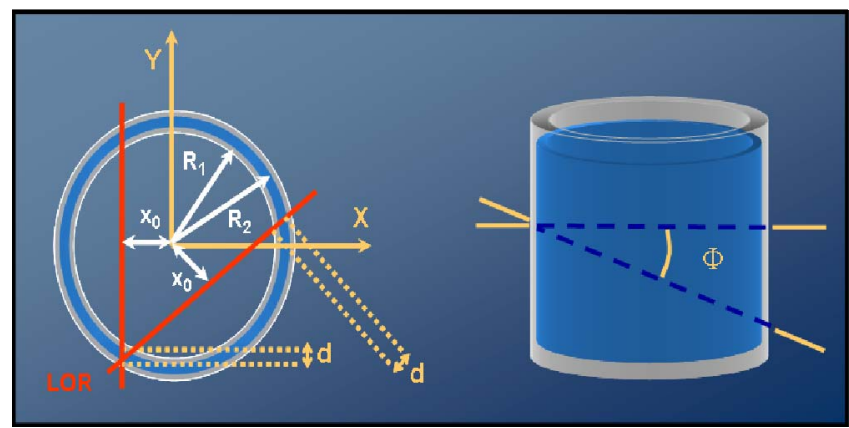

Fig. 9. Annulus phantom diagram

In 3D (Fig. 9, right):

$$
\text { Crosswise length }(3 \mathrm{D})=\frac{2}{\cos \Phi}\left\{\left(\mathrm{R}_{2}^{2}-\mathrm{x}_{0}^{2}\right)-\left(\mathrm{R}_{1}^{2}-\mathrm{x}_{0}^{2}\right)\right\}
$$

\section{REFERENCES}

[1] M. Defrise, D. W. Townsend, D. Bailey, A. Geissbuhler, C. Michel, and T. Jones, "A normalization technique for 3D PET data", Phys Med Biol, vol. 36, pp. 939-52, 1991.

[2] E.J. Hoffman, T. Guerrero, G. Germano, W.M. Digby and M. Dahlbom "PET system calibrations and corrections for quantitative and spatially accurate images", IEEE Trans. Nucl. Sci., vol 33, pp 1108-12, 1989

[3] M.E. Casey, H. Gadagkar and D. Newport, "A component based method for normalization in volume PET", Proc. 3rd Int. Meeting on Fully Three-Dimensional Image Reconstruction in Radiology and Nuclear Medicin, pp 67-71, 1995
[4] R D Badawi and P K Marsden, "Developments in component-based normalization for 3D PET", Phys. Med. Biol, vol. 44, pp 571-594, 1999.

[5] F. Hermansen, T. J. Spinks, P. G. Camici, and A. A. Lammertsma, "Calculation of single detector efficiencies and extension of the normalization sinogram in PET", Phys Med Biol, vol. 42, pp. 1143-54, 1997.

[6] B. Bai, Q. Li, C.H. Holdsworth, E. Asma, Y.C. Tai, A. Chatziioannou and R.M. Leahy, "Model-based normalization for iterative 3D PET image reconstruction", Phys. Med. Biol. vol 47, pp 2773-2784, 2002.

[7] J. J. Vaquero, E. Lage, L. Ricon, M. Abella, E. Vicente and M. Desco, "rPET Detectors Design and Data Processing", IEEE Nuclear Science Symposium Conference Record (CD-ROM), pp 2885-2889, 2005.

[8] S. Jan et al. "GATE: a simulation toolkit for PET and SPECT", Phys Med Biol, vol. 49, pp. 4543-61, 2004. 